\title{
Analysis of Surface Structures in Ru Nanocatalysts
}

\author{
N.P. Walker, B.K. Miller, and P.A. Crozier \\ School for Engineering of Matter, Transport and Energy, Arizona State University, Tempe, AZ \\ 85287-6106
}

Hydrogen fuel cells are poisoned by even small concentrations of carbon monoxide (CO). Ruthenium $(\mathrm{Ru})$ nanocatalysts are a promising candidate for converting $\mathrm{CO}$ into carbon dioxide $\left(\mathrm{CO}_{2}\right)$, in these fuel cell feedstocks. In order to have a full understanding of ruthenium's capabilities as a $\mathrm{CO}$ oxidation catalyst, the surface structure of particles in transmission electron microscope (TEM) images was analyzed via image simulations. Analyzing the surface structure begins with the fundamental principle of Wulff constructions. Wulff constructions are characteristic, three-dimensional shapes determined by the space group of the crystal and the surface energies [1]. The Wulff construction of a small Ru particle is shown in Figure 1.

At elevated temperatures in the environmental TEM, we have found that most crystalline nanoparticles observed appear to match the known topology and structure of the Wulff construction. However, to identify individual surfaces, the orientation must also be found. Lattice spacings and angles were measured using a Fourier Transform. When this was performed on the experimental image in Figure 2, the beam direction was found to be [101]. This leads to the conclusion that the (111) and (110) surfaces are visible at the edges of the particle's image. After the particle shape and orientation were determined, a model of the Wulff construction, made in CrystalMaker, was used as the input to JEMS image simulation software to simulate TEM images of the nanoparticle. JEMS can also use the aberrations measured by the image correction software of the Titan ETEM to increase image accuracy. The simulation can be seen in Figure 2 to fit the particle with two exceptions: corners are rounded in the experimental image due to the high energy of the corner sites and part of the particle is obscured and cut off due to the amorphous silica support. More details on the technique and conditions used to obtain the experimental image are found in Miller and Crozier [2].

The surfaces identified in the experimental image can now be compared in detail to the simulation. As the simulated model does not does have any surface reconstruction, all comparisons will be with respect to the simulated bulk-terminated structure. From the measurements, it can be concluded that both the (111) and (110) surfaces experience lateral surface relaxation. We will present similar measurements of surface reconstructions while the $\mathrm{Ru}$ nanoparticles are reacting with various gaseous environments including combinations of $\mathrm{CO}, \mathrm{H}_{2}$, and $\mathrm{O}_{2}$ relevant to $\mathrm{CO}$ oxidation.

References:

[1] R.V. Zucker, et. al. Journal of Materials Science 47, (2012). p. 8290-8302.

[2] B.K. Miller, P.A. Crozier. Microscopy and Microanalysis (these proceedings)

[3] J. Gavnholt. Thesis. Technical University of Denmark, 2009.

[4] The support from National Science Foundation CBET-1134464 and the use of ETEM at John

M. Cowley Center for HR Microscopy at Arizona State University is gratefully acknowledged. 


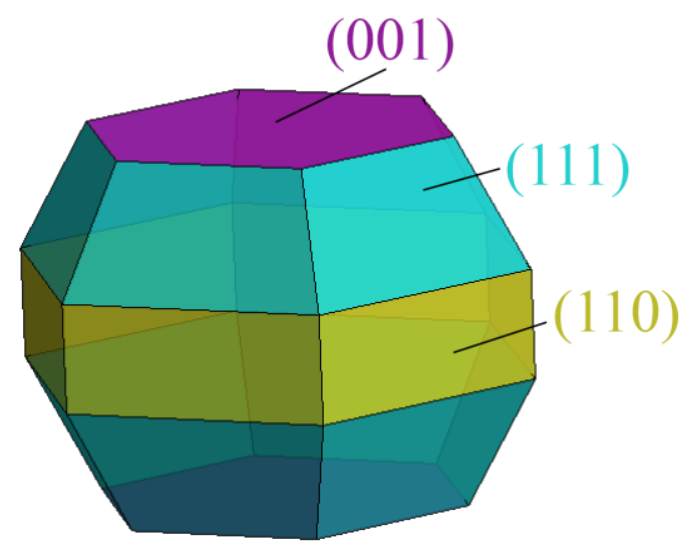

Figure 1: Wulff construction of a Ru particle which has a $\mathrm{P} 63 / \mathrm{mmc}$ space group and surface energies of 0.176 , 0.221 , and $0.221 \mathrm{eV} / \AA$ for (001), (111), and (110) respectively is made using WulffMaker [1,3].

Figure 2: Comparison between the averaged experimental TEM image and simulated Wulff construction. It can be seen that the corners are rounded due to the high energy of those sites.

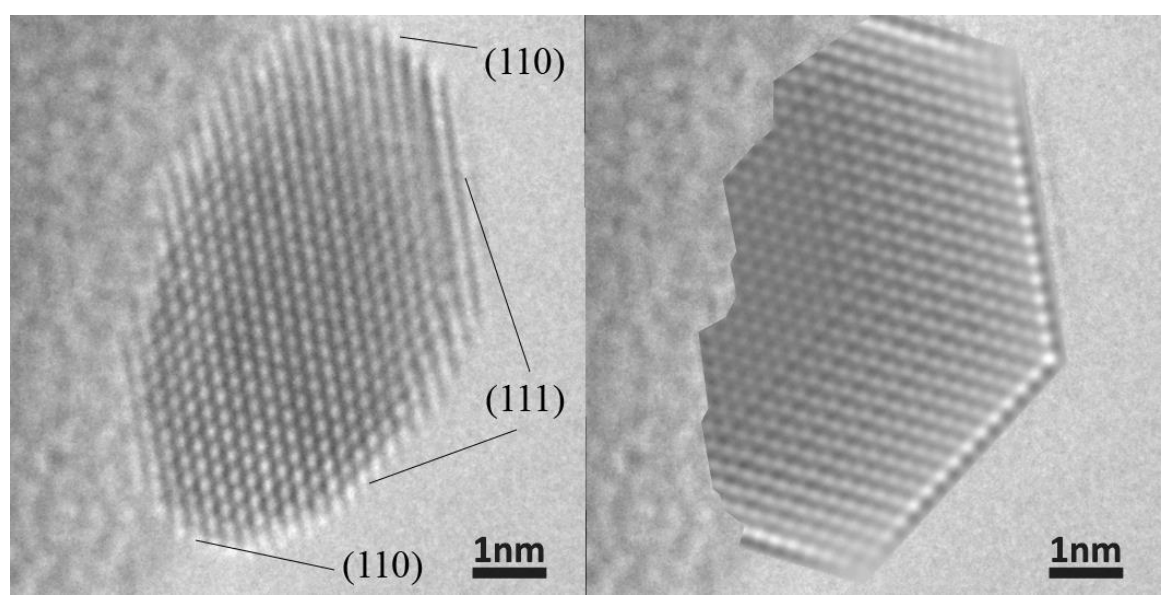

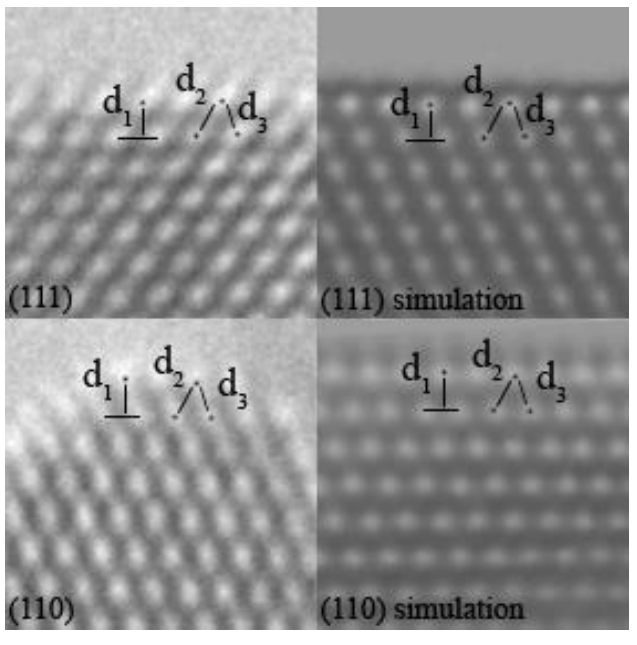

Figure 3: Comparison between surfaces found in the averaged TEM image and the bulk-terminated surface of the Wulff construction simulation. Results of measurement can be found in Table 1.
Table 1: Lattice Spacing Measurements in $\mathrm{nm}$.

\begin{tabular}{r|c|c|c}
$(111)$ & $\mathrm{d}_{1}$ & $\mathrm{~d}_{2}$ & $\mathrm{~d}_{3}$ \\
\hline Experimental & 0.27 & 0.32 & 0.25 \\
Simulation & 0.22 & 0.27 & 0.23 \\
\hline$(110)$ & $\mathrm{d}_{1}$ & $\mathrm{~d}_{2}$ & $\mathrm{~d}_{3}$ \\
\hline Experimental & 0.26 & 0.35 & 0.30 \\
Simulation & 0.25 & 0.29 & 0.27
\end{tabular}

